\title{
Applications for Advanced Solid-State Lamps
}

S ince lighting arcounts for a large fraction of electrical usage in the industrial sector, the energy efficiency of lamp systems is of major interest. One promis ng technology which has the potential for use in specialized energy-efficient lighting applications is solid-state lamp technology [1]. Recent developments in this field indicate various prospects for specialized lighting applications, such as in exit-pigns, where LEDs are now an energy-efficient, low-maintenance alternative for incandescent lamps.

There are many reasons solid-state lamps may be especially useful for new applications. They exhibit long lifetimes, on the order of 100,000 hours. Coupled with the rugged hess inherent in solid-state devices, this indicates usefulness for low maintenance applications. Fast response times also make them ideal for some applications; one recent utilization is in high-speed autombbile brake lights.

There have been several reviews of solid-state lamp applications in the telecommunications and display fields [2]-[8], but no previous survey of these devices with a perspective on niche applications where they could constitute possible replacements for conventional lamps. This review surveys the state-of-the-art n solid-state semiconductor lamp technology, with a focus on the potential for such applications, and on energy efficiency and brightness considerations.

\section{Dverview}

The terms "solid-state lamp" or "semiconductor lamp" refer to light sources based on semiconductor materials III-V ccmpounds such as gallium arsenide (GaAs) and gallium aluminum arsenide (GaAlAs), or II-VI compounds such as zinc selenide (ZnSe). There are several types.

\section{Types of Solid-State Light Sources}

1) Light-emitting diodes: The most common solidstate lamps are light-emitting diodes (LEDs) [9], [10]. They operate at tens of milliamps and a few volts, and have response times of roughly hundreds of nanoseconds. This makes LEDs compatible with integrated circuits, and ideal for use as status indicators. In illumination-type applications, however, common LEDs display some drawbacks. First, while the light is proportional to the current at low values, at higher values the intensity saturates, defining a threshold above which the process is inefficient. Second, an LED radiates brightly over only a narrow solid angle, a few tens of degrees. Third, single LEDs emit light over only a narrow spectral range. Fourth, LEDs based on technology from the 1970 s are still widely available; their low-rated performance has led to poor perception of solid-state lamps in general. However, recent brightness and efficiency gains have led to devices comparing favorably with the performance of some conventional lamp systems. New manufacturing methods, materials and ultra-large scale processing signal a possibility for lower costs, contributing to a resurgence of interest in LEDs. An example of this is the replacement of conventional bulb-reflector systems in some automobile brake lamps with LEDs or laser-diffuser systems [11].

2) Diode lasers: Under optical resonance conditions, solid-state light devices may emit coherent light [12], in which the output is extremely monochromatic, collimated and in-phase. These devices are called diode lasers, and are used in digital signal storage and retrieval, such as for optical discs, and in telecommunications. While the output of diode lasers is typically higher than in LEDs, the emitted light is coherent, and highly directional. In addi-

This article appeared in its original form at the 1993 IEEE-IAS Annual Meeting in Toronto, Ontario, Canada. Peralta, an IEEE and IA' Member, is with Ontario Hydro's W.P. Dobson Research and Development Laboratories, in Toronto. Ruda, an IEE E Member, is a Professor in the.Department of Metallurgy and Materials Science at the University of Toronto. 
tion, the bulk of commercial laser diodes radiate in the infrared (IR).

3) Superluminescent diodes: Recently, a light source with properties intermediate between laser diodes and LEDs. $\{13],[14\}$ has been demonstrated. These devices are called superluminescent diodes (SLDs). Fabrication methods are similar to those for diode lasers; an added step "spoils" the process leading to laser action, yielding highoutput high-efficiency devices with incoherent, narrowband emission. SLDs were developed for sensor and printing applications, but their high power output and efficiency, and low coherence make them good candidates for use in lighting applications. SLDs, however, are not yet widely available commercially.

4) Electroluminescent devices: Electroluminescent (EL) lamps consist of a thin-film or powder phosphor sandwiched between two electrodes. Under an applied electric field, the phosphor fluoresces, and light is emitted through the transparent front electrode [15]. Most phosphor systems involve a $\mathrm{ZnS}$ host and an orange activator, $\mathrm{Mn}$. Alternatives include $\mathrm{Ce}$ and $\mathrm{TbF}_{3}$ for green, and Pr for white light. EL devices are found in computer display screens; recently, panels have been used as backlighting for exit signs. EL sources are relatively inexpensive, but are relatively inefficient and dim. Their luminance may be increased by raising the operating voltage, but the lifetime then drops drastically.

\section{Operating Characteristics}

It should be noted that conventional light soutces function either as sources to be seen, or sources to illuminate other objects. Applications where solid-state lamps may excel are likely those in which they function as sources to be seen. Some characteristics of solid-state lamps-poor color rendering of objects, narrow output cones, lower total lumen outputs - make them impractical for general illumination.

Compactness, robustness, safety and response time are the rationale for the use of solid-state lamps in specific applications such as in instrument status indicators. However, their utility depends also on lamp efficiency, brightness, spectral output, lifetime and cost, as well as user acceptance.

1) Efficiency: In comparing solid-state light sources with conventional sources, we should note that the luminous efficiency of various lamp sources can vary between lamps and over the entire spectral range. Incandescent lamps, for example, convert only about $10 \%$ of the electric power input to the lamp to light. $90 \%$ of the radiant energy conversion is to heat, with a small amount to UV radiation [16].

Commercial LEDs are rated with efficiencies of 2-4\%. However, high-efficiency visible LEDs have been reported with efficiencies which exceed $15 \%$

\section{Poor color rendering of objects, narrow output cones, and lower total lumen outputs make solid-state lamps impractical for general illumination.}

[17]. Efficiencies of $30 \%$ are theoretically possible, a five-fold increase in efficiency over incandescents. In comparison, fluorescents have typical efficiencies of $20 \%$ and achievable efficiencies of $27 \%$. Progress has been dramatic: solid-state efficiencies have risen several orders of magnitude from values on the order of 0.01-0.1\%. If this trend is maintained, devices with efficiencies of $>15 \%$ may be expected to be commercially available within the next few years and commonplace in 5 to 10 years.

2) Luminance: A 60-watt incandescent lamp has an average luminance ranging from approximately $3 \times 10^{4} \mathrm{~cd} / \mathrm{m}^{2}$ for a "white" bulb to $1.2 \times 10^{5} \mathrm{~cd} / \mathrm{m}^{2}$ for an inside frosted bulb [16]. In comparison, LEDs exhibiting values in excess of $10^{4} \mathrm{~cd} / \mathrm{m}^{2}$ at current densities of $10 \mathrm{~A} / \mathrm{cm}^{2}$ are readily available. At an efficiency of $2 \%$, the output of solid-state lamps is of the order of $0.01 \mathrm{~cd}$, over a $25^{\circ}$ cone. Over a wider viewing angle $\left(70^{\circ}\right)$, this drops to 0.1 $\mathrm{mcd}$. Since the chip used in such lamps is from 0.25 to $1 \mathrm{~mm}$ square in size, a typical luminance is $1.6 \mathrm{x}$ $10^{4} \mathrm{~cd} / \mathrm{m}^{2}$ over a $70^{\circ}$ viewing angle. The brightness of solid-state devices is roughly proportional to the efficiency. Thus, for a lamp with an efficiency of $4 \%$, the luminance over $70^{\circ}$ is $3.2 \times 10^{4}$ $\mathrm{cd} / \mathrm{m}^{2}$. Efficiencies of $4 \%$ are well within range for LEDs, making their luminance comparable to that of a 60-watt "white" bulb incandescent. While theoretical luminances of $3 \times 10^{5} \mathrm{~cd} / \mathrm{m}^{2}$ are possible for solid-state sources, experimental lamps of $15 \%$ efficiency do exist; these have luminances of $1.2 \times 10^{5} \mathrm{~cd} / \mathrm{m}^{2}$, making these lamps as bright as incandescent light sources.

3) Spectral output: Solid-state lamp emission has a spectral width of a few hundred nanometers. In comparison, incandescent lamps emit broadband radiation peaking in the IR and trailing into the $\mathrm{far}$ IR. Only $10 \%$ of the emission is in the visible spectrum ( $400-750 \mathrm{~nm}$ ). Other broadband light sources do emit much more in the visible; for example, around $20 \%$ of a fluorescent larip's emission is in the visible. Coverage of the visible spectral range for white light illumination is not feasible for single-element solid-state devices, but may be accomplished by using large arrays of single-color diodes. Alternatively, clusters of multicolored LEDs may be used to simulate a single wideband source. 


\begin{tabular}{|c|c|c|c|}
\hline & $\begin{array}{l}\text { Incandescent } \\
\text { Lamp }\end{array}$ & $\begin{array}{l}\text { Solid-State } \\
\text { Lamp } \\
\text { (commercial) }\end{array}$ & $\begin{array}{l}\text { Solid-State } \\
\text { Lamp } \\
\text { (experimental) }\end{array}$ \\
\hline Efficiency $(\%)$ & 6 & 4 & $15-30$ \\
\hline Luminance $\left(\mathrm{cd} / \mathrm{m}^{2}\right)$ & $10^{5}$ & $10^{4}$ & $.10^{5}$ \\
\hline Spectral Output & Broadband & Narrowband & $\begin{array}{l}\text { Both narrow- } \\
\text { band and } \\
\text { broadband }\end{array}$ \\
\hline Lifetime (hours) & $10^{3}$ & $10^{5}$ & $10^{6}$ \\
\hline Estimated Unit Cost & 0.16 & 16 & presently. $10 \mathrm{c}$ \\
\hline
\end{tabular}

4) Lamp lifetime: General-use incandescent lamps have lifetimes of from 750 to 1000 hours. While some lamps are available at 2500 hours lifetime, and special-pu rpose lamps may achieve up to $10^{6}$ hours, these liffetimes are achieved at reduced luminous efficiencies [16]. For fluorescent lamps, lifetimes of $10^{5}$ hours are not uncommon. In comparison, typical lamp lifetimes for commerciallyavailable general plirpose LEDs range up to $10^{6}$ hours. Special-purpose LEDs have even longer rated lifetimes (at a higher cost), but with minimal performance detericration.

5) Manufacturin cost: Currently, the LED industry is focused on applications in telecommunications; the annual volume is estimated to be on the order of $\$ 200 \mathrm{M}[1]$, with production estimated at about $5 \times 10^{9}$ unjts and unit cost at about 1.5 to 2.0 cents. Volume sales are about 3.0 to 5.0 cents per unit. Present prpduction of materials for solidstate lamps tops over $1000 \mathrm{~m}^{2} /$ year-an immense scale, considering each unit requires only about 1 $\mathrm{mm}$ square. The unit cost for incandescents is estimated at 2.0 to 2.5 cents, with volume sales at about 6.0 cents per unit.

The primary reason this has not translated into more widespread use of LEDs is that ordi-

Unit costs of solid-state lamps,
especially LED|s, are expected to fall by
at least an order of magnitude in the
next few years.

nary incandescethts are typically 10 times brighter than cheap commercial LEDs. This fact leads to an unfavorable unit cost/performance ratio for LEDs orer incandescent sources. Obviously, a reduction in manufacturing costs is necessary to offset this disadvantage.

Two factors in cost reduction are materials and processing; each is theginning to be addressed. Bulk inorganic and organic semiconductor materials such as silicon, germanium and polymers are now being considered for use in solid-state lamps. In addition, the net cost of finished devices is tied up to the processing technique-whether devices are manufactured using vapor, liquid, or molecular beam techniques, cr other means. Work continues to develop more cost effective processing methods.

Manufacturing is also moving from the intensive single-device towards more use of integrated circuit techniques. One milestone is the achievement of a technique for building 20,000 diode lasers on a $5 \mathrm{~cm}$ diameter semiconductor wafer.

While wafer-scale production has been used in the LED field, the complexity of laser diodes has previously resulted in expensive and time-consuming production. Since SLDs are similarly complicated to produce, full-wafer techniques may be a turning point in translating SLDs to commercial usage.

With these considerations, unit costs of solidstate lamps, especially LEDs, are expected to fall by at least an order of magnitude in the next few years. Commercial introduction of SLDs is also anticipated, and these two events are expected to springboard solid-state lamps into further applications.

\section{Potential for Development}

The limitations of commercial LEDs do not preclude their use in niche applications. In fact, solidstate lamps are already available to replace $5-28 \mathrm{~V}$ miniature incandescent lamps in status indicators and appliance lamp applications [18]. While the solid-state lamps are more expensive, they exhibit enhanced energy-efficiency, longer operating lifetimes, and lower maintenance costs.

Table 1 summarizes some characteristics of solidstate lamps, in comparison to incandescents, to help identify some of the potential for improvement.

\section{Applications}

Solid-state lamps cannot compete against highintensity lamps such as sodium and high-intensity discharge. However, solid-state lamps are compact, robust, use a tenth the power of incandescents, produce no heat and are long-lived. As industry produces less expensive, brighter and more efficient models, we may expect solid-state lamps to make inroads in some niche applications now dominated by incandescent, and perhaps even fluorescent, lamps.

\section{Illuminated Signs}

Illuminated signs may be floodlit, backlit (internally-illuminated), or element-lit. Floodlit signs depend on area and brightness for attention, and thus require uniform luminance over a large area [16]. While the inhomogeneous output of LEDs over wide solid angles makes it unattractive 
for floodlit applications, LEDs are ideal in backlit or element-lit signs.

1) Internally-illuminated signs: In this type of sign a printed translucent panel is backlit by an internal light source. Examples include signs identifying stores, cinema billboards, and some posters in malls and subways. Light sources include fluorescent lamps, diffused incandescent and mercury lamps and luminous (neon-type) tube lamps. Typical luminances required are 70 to $350 \mathrm{~cd} / \mathrm{m}^{2}$ for ordinary signs, 250 to $500 \mathrm{~cd} / \mathrm{m}^{2}$ for brighter signs located in malls, 700 to $1000 \mathrm{~cd} / \mathrm{m}^{2}$ for commercial signs, 1000 to $1400 \mathrm{~cd} / \mathrm{m}^{2}$ for areas of high sign competition, and 1400 to $1700 \mathrm{~cd} / \mathrm{m}^{2}$ for emergency traffic control conditions $\{16\}$.

\section{Applications already utilizing LEDs include element-lit bouse numbers, scoreboards in sports venues and tunnel signs.

Solid-state lamps with $2 \%$ efficiencies have luminous intensities of roughly $0.01 \mathrm{~cd}$ for a $1 \mathrm{~mm}$ square device. A square meter area could be covered by one million devices, to a total luminance of $10^{4}$ $\mathrm{cd} / \mathrm{m}^{2}$, adequate to cover all cases above. This refers to red LED lamps; best applications are thus those which are satisfactory backlit with red light (e.g., warning signs).

"White" light from LEDs requires clustered red, green and blue lamps. Ultrabright LEDs now have luminous intensities which range from 0.2 (green to red) to $15 \mathrm{~cd}$ (red only, at present). This implies, for $0.1 \mathrm{~cd}$ devices, a luminance of $2 \times 10^{5}$ $\mathrm{cd} / \mathrm{m}^{2}$ for green to red ultrabright LEDs. Blue LEDs now have luminous intensities of $0.02 \mathrm{~cd}$, and luminances of $2 \times 10^{4} \mathrm{~cd} / \mathrm{m}^{2}$. At present the unit costs of such devices are high - from $\$ 0.10$ to $\$ 1$ per unit, depending on ourput.

Power usage of a backlit sign depends on the application, and it is difficult to compare systems. A sign lit by a bank of six $1.06 \mathrm{~m}$ (42") fluorescent tubes requires approximately $300 \mathrm{~W}$, including ballast, with a mean replacement time of 20,000 hours. The same sign could be backlit by a diffused bank of forty long-life 60-W incandescent lamps requiring $2400 \mathrm{~W}$, requiring replacement after about 10,000 hours. An array of 3000 individual solidstate lamps would be rated as a $300 \mathrm{~W}$ system, with a mean replacement time of 50,000 hours. These values will vary depending on the specific application.

2) Element-lit signs: These include applications where lamps form alphanumeric or graphic elements. Examples are neon-type advertising and message signs. Unlike backlit signs, where uniform monochromatic illumination is ideal, element-lit signs make use of color and apparent motion (flashing lights). For low-brightness areas, incandescents up to $11-\mathrm{W}$ are utilized; for medium-brightness areas, 25-W; and for bright areas, up to $40-\mathrm{W}$ [16]. Proportional wattages balance the effect for colored bulbs: clear 10-W, yellow $10-\mathrm{W}$, orange $15-\mathrm{W}$, red $25-\mathrm{W}$, green 25-W and blue 40-W. Motion effects--running borders and flashing signs-usually use 20-W plasma lamps, which require 0.05 to $3 \mathrm{~W}$, and have efficacies up to $0.3 \mathrm{~lm} / \mathrm{W}$ and lifetimes of 20,000 hours.

Solid-state lamps are good candidates in these applications. They exhibit lower power requirements and are longer-lived than incandescents; LED power requirements are similar to glow lamps, are 10-100 times brighter. The drawback is unit cost - the larger the sign is, the more LEDs are needed, and the higher the system cost. It is thus to be expected that the first impact will be small scale signs, such as exit-signs. Other applications already utilizing LEDs include elementlit house numbers, scoreboards in sports venues and tunnel signs.

\section{Exit-Signs}

Exit-signs are discussed separately because, apart from automobile brake lamps, they constitute one of the most important applications and markets at present for LEDs. Exit-signs are internally- or elementlit, and consist of a single or double-faced fixture. Primary light sources are incandescents, compact fluorescents and tritium lamps. Recently, however, both LED and EL-based exit-signs have come into use, in both internally-lit and element-lit forms. While the present market share of solid-state exit-signs is small, their advantages in terms of long life, low mainte-

\begin{tabular}{|c|c|c|c|c|c|}
\hline & LED & Incandescent & Compact Fluorescent & EL & Tritium \\
\hline Lamp + Fixture Cost $(\$)$ & $90-150$ & $30-130$ & $120-300$ & $180-300$ & 250-300 \\
\hline Lamp Life (years) & $15-25$ & $0.1-1$ & $1-2$ & $5-10$ & 10.20 \\
\hline Power Cons. (W) & $1-10$ & $30-50$ & $12-20$ & $0.5-1$ & 0 \\
\hline Letfer Illum. & uniform & nonuniform & nonuniform & uniform & uniform \\
\hline Relative Lumin. & medium-high & high & high & low & medium-high \\
\hline
\end{tabular}




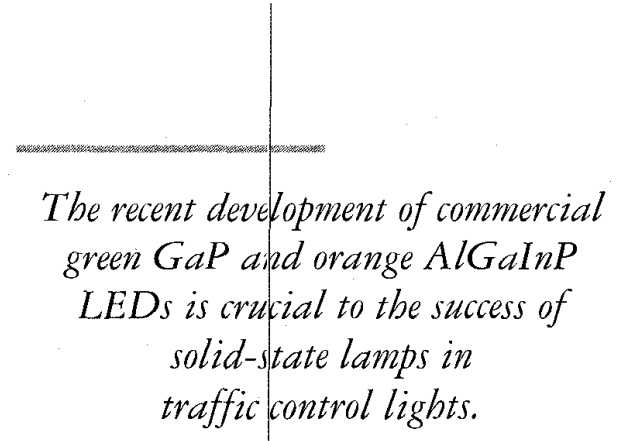

nance and reasonable cost make them attractive. Table 2 compares typlical characteristics of commercial exit-signs based on LED, incandescent, compact fluorescent, EL and tritium lamps.

LED signs are typically element-lit. Incandescent signs usually haye two 15 to 25 watt bulbs and fluorescents two 5, 7 or 9 watt lamps. EL signs are typically internally lit, and both low and high current models exist. Tritium signs utilize about 25 curies, equivalent to $2.5 \mathrm{mg}$ of tritium gas.

Both EL-based and tritium-based exit-signs consume less power than LED exit-signs. However, EL-based exit-signs are much dimmer. The U.S. National Fire Protection Association requires that internally-lit exit-signs have a luminance equal to an externa.ly-lit sign "...illuminated by not less than 5 footclandles $(54 \mathrm{~lx})$." This requirement limits the application of EL lamps, which are up to an order of magnitude dimmer, and at present attain the luminance only at a decreased lifetime. LED signs, on the other hand, comply with the brightness specification without compromising lifetime or cost. In contrast, the long life and reliability in power outage situations for tritium lamps makes them the choice of fire safety professionals. However, their use is not widespread because of ambiguous public reaction.

Because of the lirnited off-axis visibility of LED signs, some designs incorporate an additional LED stripe on the sides of the exit-sign. Some signs also exhibit LED-edgelit: panels, providing a more homogeneous light output and better visibility from off-axis angles.

\section{Traffic Control Laghts}

Lamps used in traffic applications require red, green and amber oulput. Thus, the recent development of commercial green GaP and orange AlGaInP LEDs is cruclial to the success of solid-state lamps in this application.

1) Traffic signal lamps: These consist of rugged, long-life (2000-8000 hours) 60-165 W incandescent lamps housed in a fixture containing a reflector and a circular aperture and lens plate in an appropriate color (green, amber or red). Inicial average lumens range from 675 to 2250 lumens. From geometric considerations, solid-state signal lamps would requite a cluster of some 200 LEDs each of red, amber and green for a single three-light system, or 200 multicolor LEDs. The total power dissipated would be roughly 10 watts, assuming the luminance of green LEDs can be increased to that of red LEDs, at similar power requirements. Even if green LEDs consumed ten times more power than red LEDs, the total power dissipation would be about 40 watts

A three-light traffic signal based on 60-W incandescents would consume 60 watts. If the $40-W$ LED system described above were available, it would represent a difference of $47 \mathrm{~W}$ per system. This estimate would, of course, be revised by actual performance ratings for green LEDs. One can also envision a hybrid system, in which an incandescent is used for the green signal, and solid-state lamps for red and amber signals. Such a hybrid system would consume approximately 29 watts at any given time, a $50 \%$ electrical advantage.

2) Directional arrow signals: Arrow signals consist of long-life 60- to 165 -W incandescent filament lamps behind green and amber filters and a die-cut arrow mask. A solid-state system requires development of green LEDs with ratings higher than that available now. However, the potential unit savings would be greater than for traffic signal lamps, because conventional arrow signals are internally lit, and use 60- to $165-\mathrm{W}$ incandescents. LED arrow signals would be element-lit, and require fewer LEDs than for a traffic signal lamp. From size considerations and the same assumptions above, one unit would require 40 LEDs, with a power usage of $1 \mathrm{~W}$.

3) Pedestrian signals: These are internally illuminated signs with green (or white) "WALK" and red (or white) "DON'T WALK" or "walking person" pictogram masks. All use incandescent lamps, from 60 to $100 \mathrm{~W}$ each. Since the pictogram versions are more universal, these are the versions which have been developed first for solid-state systems. Between 90 to 100 green or red LEDs outline the figure, and the whole fixture is rated at approximately 1 to $5 \mathrm{~W}$, with an electrical benefit similar to that of directional arrow signals.

Because pedestrian lights must be visible over distances shorter than for traffic signal lamps, luminance requirements are less stringent. In addition, the $30-70^{\circ}$ field of view of most LED lamps is adequate for this application. This has made LEDbased systems attractive, and LED versions are beginning to be tested in various areas [19].

4) Flashing warning beacons: This includes red and amber railway lights and overhead pedestrian crosswalk lights. A particular advantage is that the colors required are those for which commercially-available LEDs perform satisfactorily. Train warning lamps, located before a railway crossing, use rugged $75-\mathrm{W}$ incandescent lamps, rated at 500 to 1000 hours. LED replacements indicate potential savings in power consumption, maintenance and lamp replacement costs. Train warning lamps are not continuously operational; the benefit to both railways 
If the 20,000 bulbs on the Christmas tree at Rockefeller Plaza were replaced by four-bulb LED clusters, electrical costs would drop to about $\$ 1$ per bour

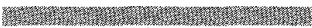

and utilities may be less than in other more appropriate circumstances. Pedestrian crosswalk signals use the same type of lamps as do railway crossings, but operate continuously. In this situation the cost savings of LED-based systems would be greater.

5) "Intelligent" bighway information signs: Highway signs give such information as distances to various destinations and exit ramp locations. These are traditionally externally-illuminated, using high-contrast painted signs with floodlight illumination. Lighting includes incandescent, tungsten-halogen, mercury, metal halide and fluorescent systems.

Solid-state lamps are not able to compete with floodlighting. Recently, however, "intelligent" highway information signs have come into use in some cities. These are comprised of arrays of gas glow or incandescent lamps. Individual lamps may be energized to form graphic or alphanumeric characters giving such information as roadwork, accidents, and other facts which, unlike destination distances, are changeable. The lamps must withstand adverse weather conditions, have fast switching rates, be compact, and consume as little power as possible. These requirements make LEDs ideal; additionally, LEDs' are compatible with the integrated circuitry driving these signs. However, "intelligent" signs require high luminance operation for visibility at long distances, pointing to a need for further developments in ultrabright LED lamps.

\section{Airport Runway Lighting}

Lighting for airport runway markers have the following requirements: low intensity (up to $10 \mathrm{~cd}$ ), medium intensity (around $1500 \mathrm{~cd}$ ) and high intensity (greater than $10,000 \mathrm{~cd}$ ). The latter two are recommended for poor visibility conditions. These considerations render LEDs usable only in the low-intensity requirements. Taxiway edge and centerline lights are typically blue $2 \mathrm{~cd}$ lamps, or $20 \mathrm{~cd}$ green, respectively. The color requirements differentiate between airport and highway traffic control. Here, the light level requirements are one order of magnitude higher than available solidstate devices in the green or blue. However, if this light level can be reached, the long-life, lowmaintenance qualities of blue and gteen LEDs qualify them for these applications in remote sites.

\section{Decorative Purposes}

This category includes such applications as seasonal holiday lighting, store displays and amusement parks. Solid-state lamps are ideal for these purposes, even relatively low-performance types, because-although the lamps must be bright enough to be seen against their backgrounds - the primary concern of these applications is not illumination.

As an example, consider Christmas lighting. As part of a demonstration, the Lighting Research Center [20] at Rensselaer Polytechnic Institute used four-bulb clusters of red and green LEDs to light an outdoor tree, in place of individual 7-W C-7 1/2 miniature incandescents. If the 20,000 bulbs on the Christmas tree at Rockefeller Plaza were replaced by such four-bulb LED clusters, the drop in electrical cost would be from $\$ 14$ to about $\$ 1$ per hour; a further cost would also be eliminated since the LED clusters could be reused year after year over their lifetime of 100,000 hours.

Other incandescents in use for outdoor decorative purposes are 5- and 10-W lamps. Here the energy savings could increase by almost $40 \%$ over the example above. A typical indoor Christmas tree system consists of a string of 100 miniature incandescents with a total power consumption of $50 \mathrm{~W}$. A string of 100 LED clusters would have dissipate 2-10 W, representing a savings of up to $48 \mathrm{~W}$ per string.

\section{Illumination for Vision Sensors}

Some automated processes employ vision systems for object detection and counting. These systems use a light source to form an image of the object onto either a single photodetector or detector array. Depending on the application, light sources used for this purpose can vary from miniature incandescent lamps to quartz halogen lamps.

Single LEDs and arrays have also been used as a cheap light source for vision systems. However, the most common LED-based systems are still based on older devices, which have low light output; many vision sensor systems must operate under low a mbient light conditions. The advent of ultrabright LED devices with outputs greater than 1 cd mean high-luminous-intensity arrays may be manufactured for operation under more general conditions. An additional feature of LED-based systems is that the single-color emission characteristic creates a high-contrast environment which is ideal for object identification through silhouetting.

\section{Spotligbting}

The relatively narrow angle of LED light output makes these devices ideal for spotlighting. While solid-state lamps cannot compete with highintensity discharge or halogen lamps for primary spotlighting, they may be useful in supplementary spotlighting roles in museum and gallery applications, where these roles are now played by incandescents. Deterioration of artwork due to heat and 
ultraviolet light from conventional sources also constitute problems that LED replacements could help alleviate.

Ultrabright red AlGaAs lamps have an output of $15 \mathrm{~cd}$ over an angle of $4^{\circ}$, while regular red lamps have an output of 1 to $5 \mathrm{~cd}$ over 7 to $15^{\circ}$. Orange LEDs have achileved $3 \mathrm{~cd}$ at $15^{\circ}$. Wider angles, up to about $70^{\circ}$ are available, with decreased luminous intensity. Fetter color-rendering may be afforded by using arrays of multicolor or whitelight LEDs.

\section{Interactive and Fast Adaptive Lighting}

The response time of solid-state lamps is fast, on the order of hundreds of nanoseconds in some cases. LEDs and laser-diffuser systems now used in brake lamps light up about 130 to 200 milliseconds faster than conventional lamps, after power application. At $100 \mathrm{~km} / \mathrm{hr}$ this represents a distance of 2.8 meters ( 9 feet). This added available stopping distance is particular y advantageous in emergency braking situations. The same principle can be used in applications with similar requirements, such as in status indicator larhps in nuclear power stations, where, despite the reliance on automatic systems, it is essential to get near-instantaneous response from status indicator lamps. At present, miniature incandescents are now in use in these applications, usually in the 5-W to 7-W range.

\section{Fiber Optics-Channeled Ligbting}

It is sometimes desirable to have a light source with no local electrical input, for safety considerations, such as for illumination in swimming pools or in hazardous environments. These needs are presently served by high-intensity discharge lamp outputs channeled through glass or plastic lightguides. More recently, fiber optics has been used because of lightness, flexibility, and cost considerations. Solid-state lamps are uniquely optimized for use in fiber optics. In addition to electrical safety, fiberchanneled LED lighting would add reliability, long life, and low maintenance costs.

\section{Discussion}

Technoeconomic Considerations

Widespread use of solid-state lamps in lighting applications will be kased on decreasing the unit cost/performance ratio. This can come about by a reduction in associated materials and manufacturing costs, by an inctease in performance parameters, or by rising sal ss volumes.

The issue of mattrials cost is addressed by the move to new materials, including silicon, germanium, and semiconclucting polymer-based lamps, which are less expensive than the usual materials. The economies of scale are also operative here. World silicon production levels are about $10^{5}$ to $10^{6} \mathrm{~m}^{2}$ year. For the past four decades, this level has increased at one order of magnitude per decade.
The production levels of III-V LED materials is about $10^{3}$ to $10^{4} \mathrm{~m}^{2} /$ year, although the year-overyear percentage increases are similar. The production levels of II-VI LED materials is roughly an order of magnitude less. At present, polymers are not used significantly in these applications, but it is sufficient to observe that polymer plants are more economic and operate at higher output levels than silicon processing plants.

The solid-state lamp industry is focused on telecommunications applications, with an annual volume of approximately $5 \times 10^{9}$ diodes/year, corresponding to a turnover on the order of $\$ 200 \mathrm{M}$ with the unit cost at about 1.5 to 2.0 cents. Volume

\section{The response time of solid-state lamps is fast, on the order of bundreds of nanoseconds in some cases.}

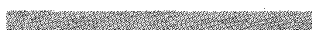

sales are about 3.0 to 5.0 cents per unit; annual growth of at least 25 percent is expected. Similar unit costs are expected for solid-state lamps.

One key to reducing costs will be in moving from a single-device approach to more widespread use of integrated circuit techniques, including wafer-scale mass production and testing. In tandem with new process techniques, this could result in lower costs, while preserving desirable performance characteristics of complex device structures. Unit costs of solid-state lamps, especially LEDs, are thus anticipated to fall by an order of magnitude in the next few years, enough to make LEDs economically competitive with incandescents.

\section{Performance Improvements}

Improvement of the unit cost/performance ratio for solid-state lamps depends also on increasing performance parameters to make them more suitable for applications. Lamp lifetimes, of from $10^{5}$ to $10^{6}$ hours, are already satisfactory for most applications; other performance parameters, such as the efficiency, luminance, and spectral and angular outpur, require some improvement.

Commercial high-efficiency LEDs have efficiencies of 3 to $8 \%$, and lab devices have shown values of $15 \%$, already challenging the efficiencies of incandescents. At specific spectral outputs, the highest values reported to date are roughly $0.8 \%$ for blue $\mathrm{GaN}$ lamps, just over $1 \%$ for green InGaAlP lamps and $15 \%$ for red GaP lamps.

Typical luminous intensities currently range from $0.05 \mathrm{~cd}$ for blue $\mathrm{SiC}$ lamps, $1.5 \mathrm{~cd}$ for green InGaAlP lamps, $3 \mathrm{~cd}$ for orange InGaAlP lamps to $15 \mathrm{~cd}$ for red AlGaAs lamps. Red SLDs have already shown luminous intensities of roughly $10 \mathrm{~cd}$ and efficiencies of $8 \%$; SLDs are still research-level 
devices, and commercial application is expected to be several years away. The brightest commercially-available green GaP LEDs achieve a luminous intensity of about $0: 8 \mathrm{~cd}$, in contrast to the InGaAlP lamp value of $1.5 \mathrm{~cd}$. Manufacturers predict that blue lamps with about $1 \mathrm{~cd}$ will soon be commercially available.

Red, orange or yellow LEDs are fairly common. Shorter waveleng th LEDs came into use in the mid to late eighties, most significantly commercially viable blue lamps, made mainly from $\mathrm{SiC}$. Other materials also demonstrated include $\mathrm{ZnSe}, \mathrm{GaN}$ and ZnS. While individual solid-state lamps are essentially single-color devices, arrays of red, green and blue LED lamps may be used to attain other colors. Using this technique, multicolor and white light operation has been shown in a device arrayed from one red $\mathrm{GaP}$, one green $\mathrm{GaP}$ and two blue $\mathrm{SiC}$ LEDs. The lamp emits a spectrum of colors from blue to red, including white.

A big limitation of LEDs is the narrow, anisotropic distribution pattern of its light output. There is very little research on tailoring this distribution to be more uniform in single devices. Instead, the tactic of manufacturers has been to create miniature clustered LEDs, with overlapping emission patterns, so that the total light output is more or less uniformly distributed. The relatively small size of the active LED element (on the order of a square millimeter) makes this solution possible.

\section{Summary}

Lamps based on solid-state semiconducting materials constitute a novel high-performance light source only recently being considered for illumination applications. Their utility is based on certain inherent advantages over conventional lamps. They can be made extremely compact, are safe and robust, have quick response times to operating current changes, and have long operational lifetimes, on the order of $10^{5}$ to $10^{6}$ hours. While commercial solid-state lamps exhibit efficiencies of 3 to $8 \%$, efficiencies of up to $15 \%$ have been attained for experimental solid-state lamps, with $30 \%$ efficiencies thought to be feasible. Luminous intensities greater than $15 \mathrm{~cd}$ have been recorded for red-emitting solid-state lamps. Utilization of new materials and integration techniques could lead to a great reduction in materials and processing cost. Furthermore, other developments in highefficiency LEDs and superluminescent diodes may lead to the brightest and most efficient solid-state light sources yet.

Currently, some characteristics of solid-state lamps-for example, the relatively low efficiencies for blue lamps, the anisotropic output distribution, and high unit costs-limit more widespread usage. If these can be addressed, solid-state lamp technology could have wide application as replacements for conventional sources in other applica- tions, as well as in the specialized applications outlined above.

\section{Acknowledgments}

Thanks are due to a number of representatives from the lighting and semiconductor industries, too many to be named individually. However, for his exceptional interest and help, we should mention Michael Ouellette of the National Research Council of Canada. This paper is based on a series of more comprehensive reports, supported by, and available through, the Canadian Electrical Association and Ontario Hydro.

\section{References}

[1] S. B. Peralta and H. E. Ruda, "Advariced Solid-State Lighting," presented at the Canadian Electrical Association (CEA) Annual Meeting, Toronto, Ontario, March 1994. (See also S. B. Peralta, "Energy-Efficient Solid-State Lighting," CEA Report 9111-UJ-850, December 1992; and S. B. Peralta and H. E. Ruda, "The Potential for Energy-Efficient Solid-State Light Sources," Ontario Hydro Research Report E91-6-K, April 1991.

[2]J. W. Allen, "Electroluminescence in semiconductors," Acta Pbysica Polonita A, vol. A77, pp. 5-16, 1990.

[3] G. Grant, "Light sources: LEDs and LDs for fiber-optic applications," Evaluation Engineering, vol. 28, pp. 171-185, 1989.

[4] P. W. Shumate, "Recent progress in long-wavelength lightwave devices," Proc. SPIE, vol. 722, pp. 230-234, 1987.

[5] R. Hall, "Semiconductor lamps," in Lamps and Lighting, S. T. Henderson and A. M. Marsden, eds., London: William Clowes, 1972.

[6] M. G. Craford, "Recent developments in light-emittingdiode technology," IEEE Trans. Electron. Dev, vol. 24, pP. 935-946, 1977

[7] R. N. Bhargava, "Recent advances in visible LEDs," IEEE Trans, Electron. Dev., vol. 22, pp. 691-701, 1975.

[8] D. G. Thomas, "Light-emitting diodes," IEEE Trans. Electron. Dev, vol. 18, p. 621-627, 1971.

59] A. A. Bergh and P. J. Dean, Light-Emitting Diodes, Oxford: Clarendon, 1976.

[10] E. W. Williams, Luminescence and the Light-Emitting Diode. Oxford: Pergamon Press, 1978

[11] G. Malloy, "Switching to LED lamps sheds light on auto safety," Toronto Siar, p. F13, March 16, 1991.

[1.2] G. Burns and M. I. Nathan, "P-n junction lasers," Proc. IEEE, vol. 52 , p. $770,1964$.

[13] T. P. Lee, C. A. Burrus and B. I. Miller, "A stripe-geometry double-heterostructure amplified-spontaneous-emission (superluminescent) siode," IEEE J. Quant. Electron, vol. 9, p. 820,1973

[1.4] K. Imanaka, K. "Cavity length dependence of optical characteristics in high power narrow stripe GaAs superluminescent diodes," IEEE Photonics Technol. Lett., vol. 2, pp. $705-707,1990$

[15] P. J. Dean, "Comparisons and contrasts between light emitting diodes and high field electroluminescent devices," J. Luminesc, vol. 23, pp. 17-53, 1981.

[16]J. E. Kaufman and H. Haynes, Eds IES Lighting Handbook, Reference Volume, Application Volume, New York: Illuminating Engineering Sociery (IES), 1984; and Application Volume, New York: IES, 1987.

[17] R. Solomon and D. DeFevere, "Efficiency shift in very high efficiency GaP ( $\mathrm{ZnO}$ ) diodes," Appl. Phys, Lett, vol. 21, pp. 257-260, 1972.

[18] Marktech International. Solid State Lamp Selector Guide 1992, New York: Marktech, 1992.

[191 J. Miller, "LEDs promise to light up furure," Toronto Star, p. B16, March 1, 1992.

[20] M. Tulloch, "Accent on applications," Photonics Spectra. January, pp. 16-18, 1992. 